\title{
The Latest Results from the Alpha Magnetic Spectrometer
}

\author{
Andrei Kounine*广 \\ Massachusetts Institute of Technology, Cambridge, USA \\ E-mail: Andrei.Kounine@cern.ch
}

The Alpha Magnetic Spectrometer (AMS) is a 15-nation project on the International Space Station (ISS). Following a 16-year period of construction and testing and a precursor flight on the Space Shuttle in 1998, AMS was installed on the ISS on May 19, 2011. AMS is a precision particle physics magnetic spectrometer. Since its installation on the ISS, it has collected more than 140 billion cosmic rays. Precision measurements by AMS of the fluxes of cosmic ray positrons, electrons, antiprotons, protons and light nuclei as well as their ratios show several unexpected and intriguing features. The new AMS results on the positron flux reveal a new source of high energy positrons. Surprisingly, in this rigidity range the spectral indices of cosmic ray nuclei experience progressive hardening over the rigidity interval of few hundred GV. AMS continues studies of complex antimatter candidates with stringent detector verification and collection of additional data.

European Physical Society Conference on High Energy Physics - EPS-HEP2019 -

10-17 July, 2019

Ghent, Belgium

\footnotetext{
* Speaker.

${ }^{\dagger}$ On Behalf of the AMS Collaboration.
} 


\section{AMS Detector}

The AMS Experiment is the most powerful physics detector of charged cosmic rays ever deployed in space. As a magnetic spectrometer, AMS is unique in its exploration of a new and exciting frontier in physics research. Following a 16-year period of construction and testing and a precursor flight on the Space Shuttle in 1998, AMS was installed on the International Space Station, ISS, on 19 May 2011 to conduct a long duration mission of fundamental physics research in space. Its main physics objectives are the understanding of dark matter and complex antimatter in the cosmos, studies of the properties of primary and secondary cosmic rays as well as the search for new, unexpected phenomena. The improvement in accuracy over previous measurements is due to its long exposure time in space, large acceptance, built in redundancy and thorough calibration.

The layout of the AMS detector [1] is shown in Fig. 1. It consists of 9 planes of precision silicon Tracker; a Transition Radiation Detector, TRD; four planes of Time of Flight counters, TOF; a Magnet; an array of anti-coincidence counters, ACC, surrounding the inner Tracker; a Ring Imaging Čerenkov detector, RICH; and an Electromagnetic Calorimeter, ECAL.

\section{Precision measurements of the positrons, electrons, and antiprotons}

Studies of light cosmic ray antimatter species, such as positrons and antiprotons are crucial for the understanding of new phenomena in the cosmos, since the yield of these particles from cosmic ray collisions with the interstellar medium is small. Our data [2] have generated widespread interest and discussions of the observed excess of high energy positrons. The explanations of these results included three classes of models: production of high energy positrons in the interactions of cosmic ray nuclei with interstellar gas [3], acceleration of positrons to high energies in astrophysical objects [4] such as pulsars, and annihilation of dark matter particles [5, 6].

Over the last fifty years, there have been many measurements of the fluxes in cosmic rays of protons, electrons, positrons, helium, and heavier nuclei. In Figure 2(left), the most recent measurements of the positron and electron fluxes before AMS [7] are presented. As seen, the

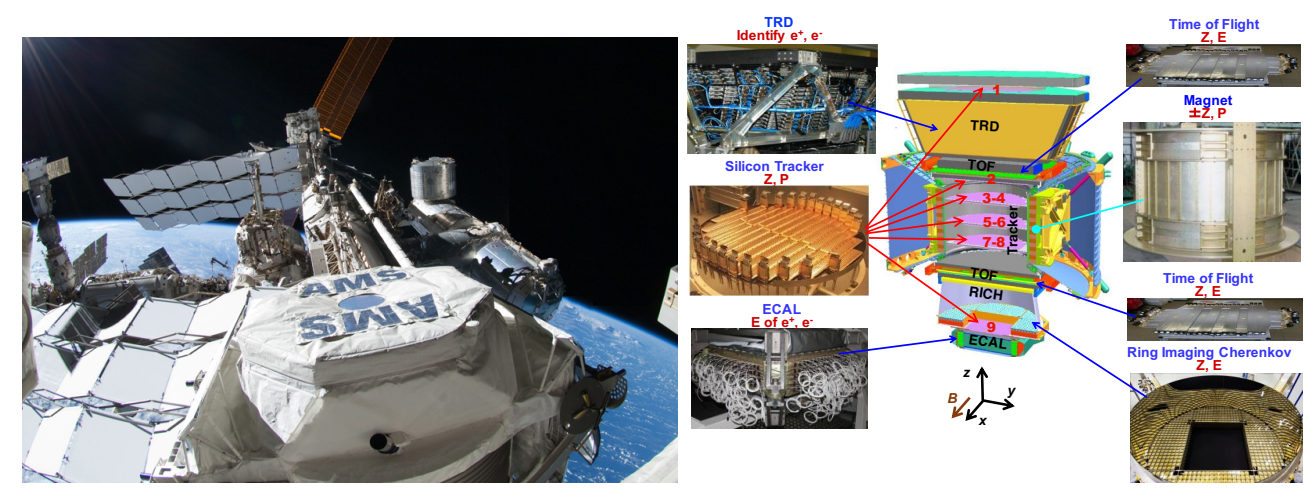

Figure 1: (left) AMS is a unique precision magnetic spectrometer on the ISS. (right) The AMS detector and its main elements. The Tracker measure the particle charge, sign and momentum. The TRD identifies electrons and positrons. The TOF and the RICH measure the charge and velocity. The ECAL independently identifies electrons and positron and measures their energy. 

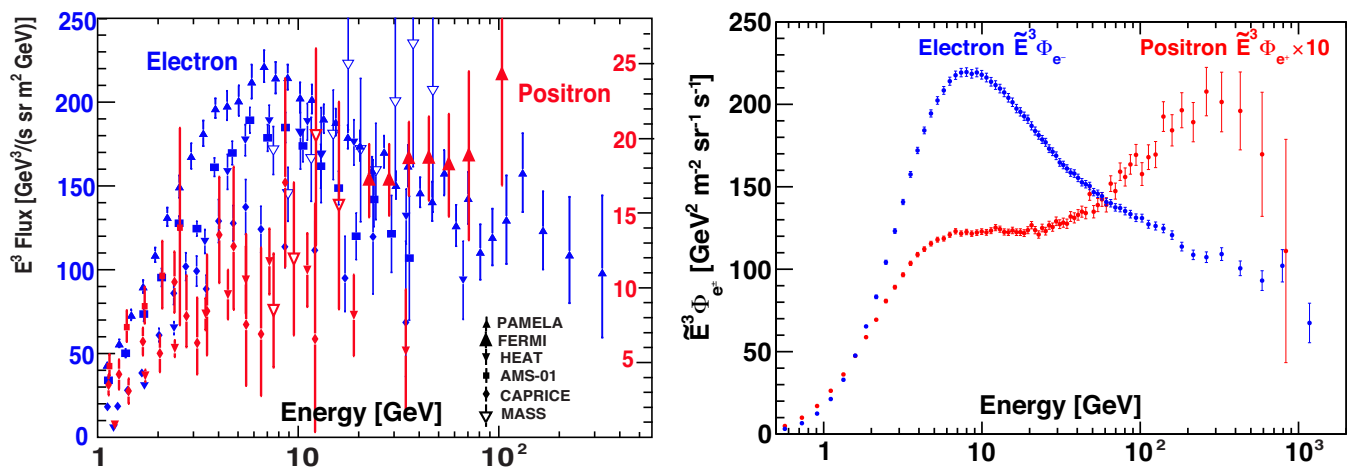

Figure 2: (left) The best measurements of the electron and positron spectra, $E^{3} \Phi_{e^{-}}$and $E^{3} \Phi_{e^{+}}$before AMS. (right)The AMS measurements of the positron and electron spectra extend the energy range to a TeV region.

data have large errors and are not always consistent with each other. AMS performed precise measurements of positron and electron fluxes up to $1.4 \mathrm{TeV}$ with on 1.9 million of positrons and 28.1 million electrons collected by AMS from May 19, 2011 to November 12, 2017 [8]. These measurements are presented in Figure 2(right). As seen, the new AMS data significantly extend the flux measurements into an uncharted high energy region with much improved precision.

We describe the behaviour of the positron flux using the simplest model, in which the positron flux is parameterized as the sum of a power law diffuse term and a source term:

$$
\Phi_{e^{+}}(E)=\frac{E^{2}}{\hat{E}^{2}}\left[C_{d}\left(\hat{E} / E_{1}\right)^{\gamma_{d}}+C_{s}\left(\hat{E} / E_{2}\right)^{\gamma_{s}} \exp \left(-\hat{E} / E_{S}\right)\right] .
$$

The fit results are presented in Figure 3(left). The diffuse term describes the low energy part of the flux dominated by the positrons produced in the collisions of ordinary cosmic rays with the interstellar gas. [9]. It gradually vanishes with increasing energy. The source term dominates the positron spectrum at high energies leading to the observed excess of the positron flux above $\sim 25 \mathrm{GeV}$. The drop-off of the flux above $284 \mathrm{GeV}$ is very well described by the sharp exponential cutoff of the source term. The significance of the source term energy cutoff is at the 99.99\% C.L.
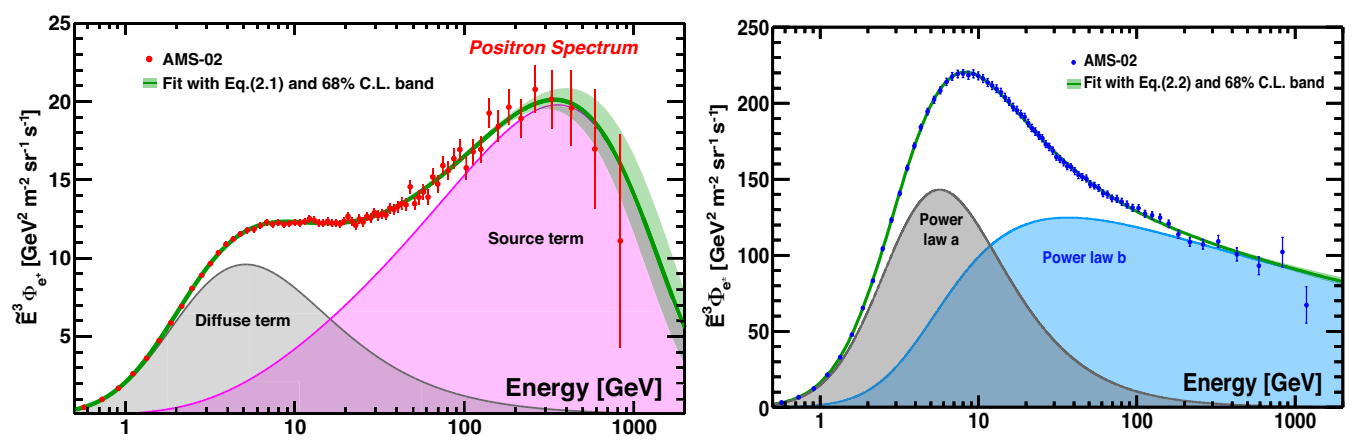

Figure 3: (left) The fit of Eq. (2.1) (green line) to the positron flux in the energy range $[0.5-1000] \mathrm{GeV}$ together with the $68 \% \mathrm{CL}$ interval (green band). (right) The two-power law fit (Eq. (2.2)) to the electron flux data in the energy range $[0.5-1400] \mathrm{GeV}$. with the $68 \%$ C.L. (green band). 

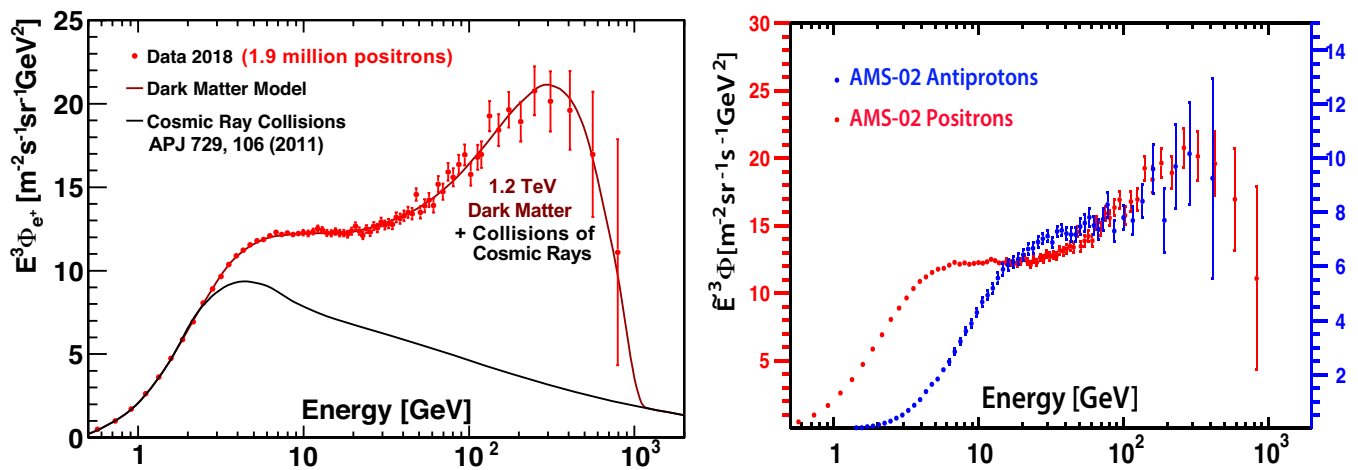

Figure 4: (left) The measured positron spectrum $\left(E^{3} \Phi_{e^{+}}\right)$in comparison with the 1.2 Tev Dark Matter model showing an excellent agreement of the model with the data. (right) Comparison of the AMS positron and antiproton spectra. As seen, at high energies, the AMS antiproton data show nearly identical energy dependence as positrons .

The electron flux shows completely different behaviour (see Figure 3(right)). It is well described by the sum of two power law components:

$$
\Phi_{e^{-}}(E)=\frac{E^{2}}{\hat{E}^{2}}\left[1+\left(\hat{E} / E_{t}\right)^{\Delta \gamma_{t}}\right]^{-1}\left[C_{a}\left(\hat{E} / E_{a}\right)^{\gamma_{a}}+C_{b}\left(\hat{E} / E_{b}\right)^{\gamma_{b}}\right]
$$

As seen in Figure 3 the electron and positron spectra have distinctly different magnitudes and energy dependences. The different behaviour of the cosmic-ray electrons and positrons measured by AMS is clear evidence of their different origins.

The characteristic behaviour of the positron spectrum shows that high energies positrons predominantly originate from new sources: either dark matter annihilation $[5,6]$ or astrophysical sources [4]. As an example, Figure 4(left) shows the comparison of AMS data with a dark matter model (based on Ref. [5]) with a mass of $1.2 \mathrm{TeV}$. This good agreement needs to be verified with more statistics at high energies, especially above $1 \mathrm{TeV}$.

We also found that the behaviour of the antiproton spectrum (based on $5.6 \times 10^{5}$ antiproton events in the absolute rigidity range $1-525 \mathrm{GV}$ ) is similar to that of positrons for energies $>60 \mathrm{GeV}$, as illustrated in Figure 4(right). The existence of the cutoff in the antiproton energy spectrum is to be expected if high energy antiprotons originate from dark matter annihilation. Note that antiprotons are not produced by pulsars. The continuation of data taking on antiprotons to 2024 and beyond will provide an important confirmation of the origin of high energy positrons and antiprotons.

Astrophysical point sources like pulsars will imprint a higher anisotropy on the arrival directions of energetic positrons than a smooth dark matter halo. If the excess of positrons has a dark matter origin, it is expected to be isotropic. The dipole anisotropy is given by $\delta=3\left(C_{1} / 4 \pi\right)^{1 / 2}$, where $C_{1}$ is the dipole moment. Limits on the amplitude of a dipole anisotropy $\delta$ (see Figure 5) in any axis in galactic coordinates in the energy range above $16 \mathrm{GeV}$ were found to be $\delta<0.019$ for positrons and $\delta<0.005$ for electrons at the 95\% C.L. [10]. Over its lifetime, AMS will reach a dipole anisotropy sensitivity for positrons of $\delta \simeq 0.01$ at the $95 \%$ C.L. 

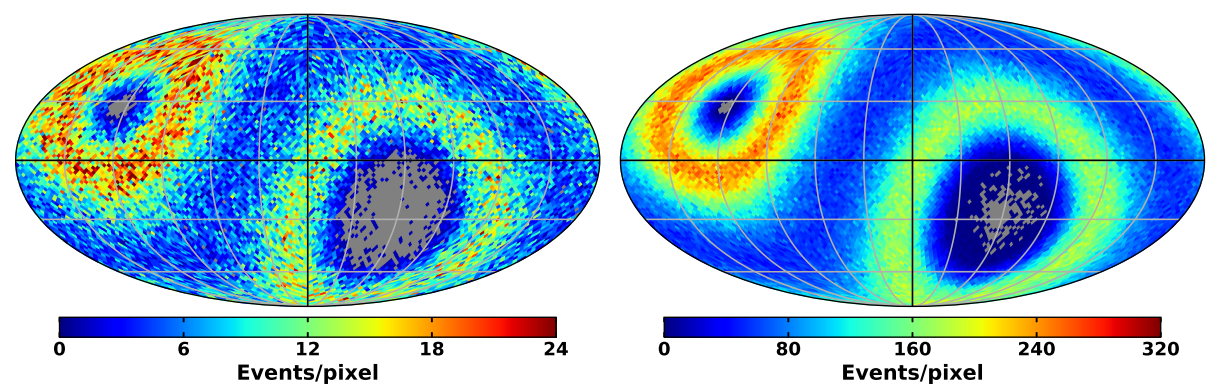

Figure 5: (left) Map of the incoming positron directions in galactic coordinates observed by AMS on the ISS. (right) Map of the incoming electron directions in galactic coordinates observed by AMS on the ISS. Both maps are consistent with the expectations for isotropic fluxes.

\section{Properties of fluxes of primary and secondary cosmic ray nuclei}

The AMS detector comprises seven instruments, which independently identify different elementary particles as well as nuclei. Protons, helium, lithium, beryllium, boron, carbon, oxygen and heavier nuclei up to iron are intensively studied by AMS (see Fig. 6(left)).

It is assumed that protons, helium, carbon and oxygen are accelerated directly by supernovae shock waves whereas lithium, beryllium and boron are produced by collisions of primary cosmic rays with the interstellar medium. Primary cosmic rays carry information about their original spectra and propagation, and secondary cosmic rays carry information about the propagation of primary and secondary cosmic rays and the interstellar medium.

High statistics, precision measurements of the helium, carbon, and oxygen fluxes [11] from $2 \mathrm{GV}$ to $3 \mathrm{TV}$, show that these fluxes all deviate from a single power law. Their spectral indices all progressively harden above $200 \mathrm{GV}$. Above $60 \mathrm{GV}$, the three fluxes have identical rigidity dependence, as illustrated in Fig.6. The secondary nuclei fluxes (lithium, beryllium, and boron) [11] also harden above $200 \mathrm{GV}$, but, as seen in Fig.6, their rigidity dependences are distinctly different com-
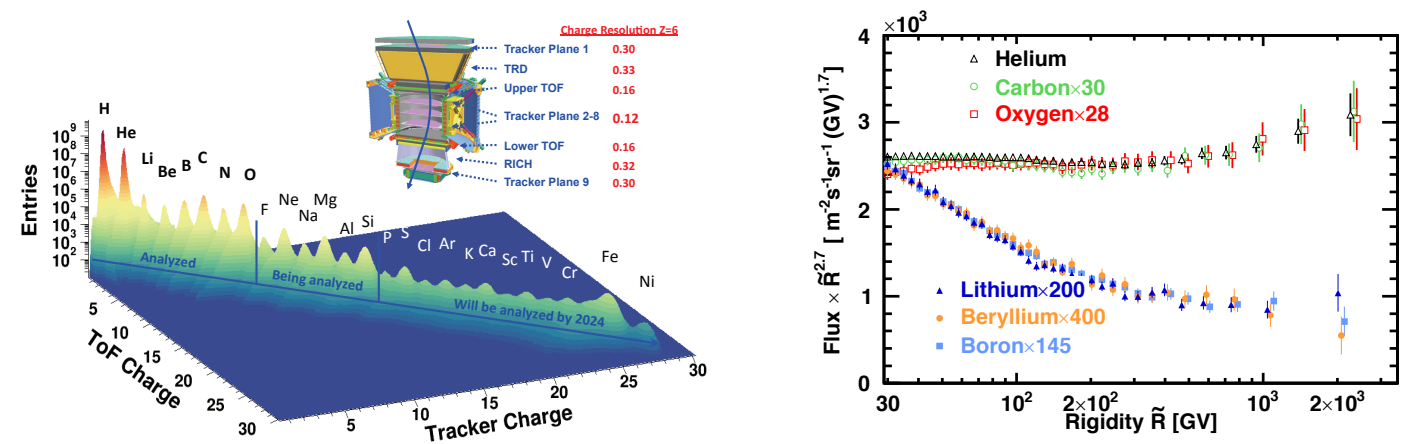

Figure 6: (left) Cosmic ray nuclei measured by AMS. As seen AMS will explore high-Z nuclei beyond Fe. (right) Comparison of the AMS primary and secondary cosmic ray fluxes multiplied by $R^{2.7}$ as a function of rigidity above $30 \mathrm{GV}$. As seen, the three primary fluxes have an identical rigidity dependence above $60 \mathrm{GV}$, as do the three secondary fluxes above $30 \mathrm{GV}$. They all experience hardening above $200 \mathrm{GV}$. However, the rigidity dependences of primary cosmic rays fluxes and of secondary cosmic rays fluxes are distinctly different. 

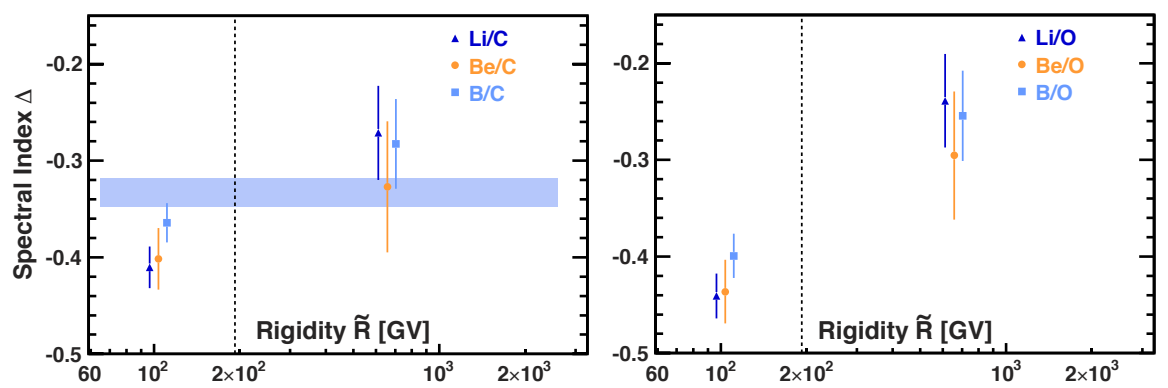

Figure 7: (left plot) The AMS secondary to primary flux ratio spectral indices $\Delta$ as functions of rigidity for $\mathrm{Li} / \mathrm{C}, \mathrm{Be} / \mathrm{C}$, and $\mathrm{B} / \mathrm{C}$. The horizontal band indicates the fit to the $\mathrm{B} / \mathrm{C}$ ratio alone. (right plot) The results for $\mathrm{Li} / \mathrm{O}, \mathrm{Be} / \mathrm{O}$, and $\mathrm{B} / \mathrm{O}$. The vertical dashed line shows the interval boundary.

pared to those of the primary cosmic rays fluxes. AMS performed unique studies in this rigidity range to understand the dynamics of the observed progressive hardening [11]. Figure 7 shows that the ratio of secondary to primary fluxes exhibits a hardening of $0.13 \pm 0.03$.

\section{Complex Antimatter in Cosmic Rays}

The Big Bang origin of the Universe requires that matter and antimatter be equally abundant in the beginning of the universe. The explanation of the absence of antimatter in a complex form requires both a strong symmetry breaking and a finite proton lifetime. Despite the outstanding experimental efforts, no evidence of strong symmetry breaking nor of proton decay have been found. Therefore, the observation of a single anti-helium event in cosmic rays is of great importance.

In eight years of operations, AMS has collected over 140 billion events. From this unparalleled sample of charged cosmic rays one billion events with charge $|Z|=2$ are selected. All these events have been identified as Helium nuclei, except few that have negative rigidity or $Z=-2$. One of these anti-He candidates is presented in Fig. 8. All of the $\mathrm{Z}=-2$ candidates have one common and peculiar feature - mass around either ${ }^{3} \mathrm{He}$ or ${ }^{4} \mathrm{He}$. At a rate of approximately one antihelium candidate per year and a required signal to background rejection of one in a billion, a detailed

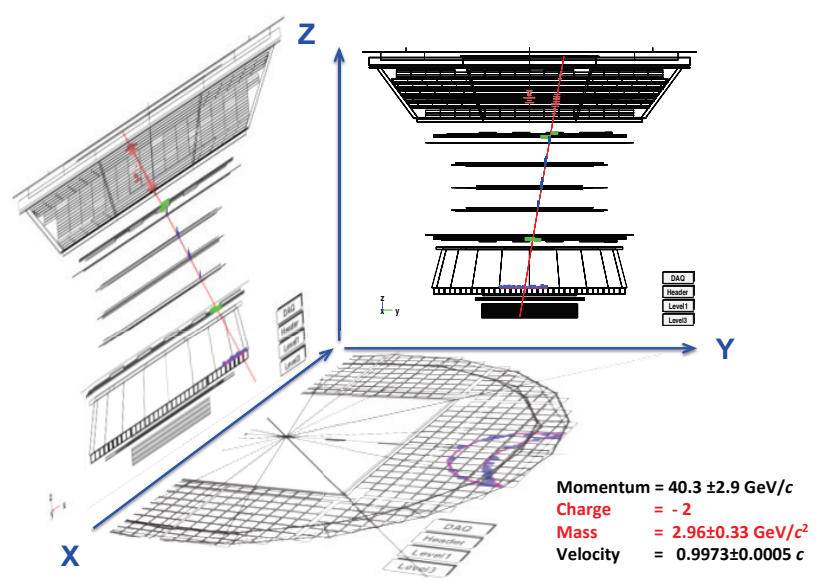

Figure 8: An antihelium candidate with a mass of ${ }^{3} \mathrm{He}$. 
understanding of the instrument is required but is exceedingly difficult and time consuming. In the coming years one of our main efforts is to perform stringent detector verification and to collect more data in order to ensure that these $Z=-2$ events are indeed anti-helium.

\section{Conclusions}

In eight years on the ISS, AMS has recorded more than 140 billion cosmic ray events. The latest AMS measurements of the cosmic ray positrons, electrons, antiprotons, protons and nuclei provide precise and unexpected information on the production, acceleration and propagation of cosmic rays. The accuracy and characteristics of the data, simultaneously from many different types of cosmic rays require the development of a comprehensive model of cosmic rays. Of particular significance is our study of complex antimatter in the cosmos. Through stringent detector verification, collecting additional data and anti-deuteron analysis we will ensure that the observed $\mathrm{Z}=-2$ events are indeed anti-helium.

As a magnetic spectrometer studying cosmic rays, AMS is unique in its precision and energy reach. For the foreseeable future this is the only magnetic spectrometer in space to perform precision measurements and to explore the unknown with high expectations for exciting discoveries.

\section{References}

[1] S.C.C. Ting, Nucl. Phys. B, 243-244 12 (2013); A. Kounine, Int. J. Mod. Phys. E 21123005 (2012) .

[2] M. Aguilar et al., Phys. Rev. Lett. 110, 141102 (2013); L. Accardo et al., Phys. Rev. Lett. 113, 121101 (2014); M. Aguilar et al., Phys. Rev. Lett. 113, 121102 (2014); M. Aguilar et al., Phys. Rev. Lett. 113, 221102 (2014); M. Aguilar et al., Phys. Rev. Lett. 117, 091103 (2016).

[3] R. Cowsik et al.,, Astrophys. J. 786, 124 (2014); K. Blum et al.,, Phys. Rev. Lett. 111, 211101 (2013).

[4] T. Linden and S. Profumo, Astrophys. J. 772, 18 (2013); P. Mertsch and S. Sarkar, Phys. Rev. D 90, 061301 (2014).

[5] J. Kopp, Phys. Rev. D 88, 076013 (2013).

[6] C.H. Chen et al., Phys. Lett. B 747, 495 (2015); H.C. Cheng et al., JCAP 03, 041 (2017); A. Cuoco et al., Phys. Rev. Lett. 118191102 (2017);

[7] O. Adriani et al., Phys. Rev. Lett. 111081102 (2013). M. Ackermann et al., Phys. Rev. Lett. 108 011103 (2012). M. Aguilar et al., Phys. Lett. B 646145 (2007). J. J. Beatty et al., Phys. Rev. Lett. 93 241102 (2004); C. Grimani et al., Astron. Astrophys. 392, 287 (2002); M. Boezio et al., Adv. Space Res. 27, 669 (2001).

[8] M. Aguilar et al., Phys. Rev. Lett. 122, 041102 (2019); M. Aguilar et al., Phys. Rev. Lett. 122, 101101 (2019); N. Zimmerman on behalf of AMS, presented at this Confefence; C. Zhang on behalf of AMS, presented at this Conference.

[9] I. V. Moskalenko and A. W. Strong, Astrophys. J. 493, 694 (1998); A. E. Vladimirov et al., Comput. Phys. Commun. 182, 1156 (2011). R. Trotta, G. Johannesson, I. Moskalenko, T. Porter, R. Ruiz de Austri, and A. Strong, Astrophys. J. 729, 106 (2011).

[10] J. Casaus on behalf of AMS, presented at this Conference.

[11] M. Aguilar et al., Phys. Rev. Lett. 119, 251101 (2017). M. Aguilar et al., Phys. Rev. Lett. 120, 021101 (2018). L. Derome on behalf of AMS, presented at this Conference. 